\title{
Aplicación de metodologías de aprendizaje activo para promover la adquisición de competencias en la asignatura Minimización y Valorización de Residuos
}

\section{Luisa María Gómez Sainero a , Elena Díaz Nieto ${ }^{b}$, Asunción Quintanilla Gómez}

Sección de Ingeniería Química, Facultad de Ciencias, Universidad Autónoma de Madrid, Cantoblanco, 28049 Madrid, Spain luisa.gomez@uam.es, $\underline{\text { belena.diaz@uam.es, }}$ a asuncion.quintanilla@uam.es

\begin{abstract}
The subject of Waste Minimization and Valorization of the Master in Chemical Engineering from the Rey Juan Carlos University and Autonomous University of Madrid (elective with reduced number of students), was designed and taught using a methodology based on active learning that has been evaluated after four years of experience. In order to achieve the acquisition of all the competences associated with the subject, and therefore better learning outcomes, a great diversity of training activities were selected, aimed at enhancing each of the basic, general, transversal and specific competences assigned. These include cooperative, collaborative, problem-based learning methodologies, case studies, flipping and networked teaching. The activities that give more prominence to the student, Case Studies and Presentation, allow to evaluate more competences, they lead to very good learning results, with high grades in the frequent evaluation (8-9,3) and favor the acquisition and integration of the knowledge thus obtaining good final grades. In addition, the methodology applied encouraged the students' commitment and motivation, with the assessment of the subjects being high and superior to the average of the master's degree and the other elective subjects.
\end{abstract}

Keywords: Active learning, problems solving, case studies, flipped learning, network teaching.

\footnotetext{
Resumen

La asignatura de Minimización y Valorización de Residuos del Máster en Ingeniería Química por la Universidad Rey Juan Carlos y Universidad Autonoma de Madrid (optativa con número de alumnos reducido), se diseñó e impartió aplicando una metodología basada en el aprendizaje activo y participativo que ha sido evaluada tras cuatro años de experiencia. Con el objeto de conseguir la adquisición de todas las competencias asociadas a la asignatura, y por ende unos mejores resultados de aprendizaje, se seleccionaron una gran diversidad de actividades formativas enfocadas a potenciar cada una de las competencias básicas, generales, transversales y específicas asignadas. Éstas incluyen metodologías de aprendizaje colaborativo, cooperativo, basado en problemas, en casos de estudio, aula
} 
inversa y docencia en red. Las actividades que dan más protagonismo al estudiante, Casos de Estudio y Presentación, permiten evaluar más competencias, conducen a muy buenos resultados de aprendizaje, con altas calificaciones en la evaluación frecuente (8-9,3) y favorecen la adquisición e integración de los conocimientos lo que se traduce en buenas calificaciones finales. Además la metodología aplicada fomentó el compromiso y motivación de los estudiantes siendo la valoración de la asignatura por parte de los mismos alta y superior a la media del máster y de las otras asignaturas optativas.

Palabras clave: Aprendizaje activo, resolución de problemas, estudio de casos, aula inversa, docencia en red.

\section{Introducción}

Una de las principales funciones de la Universidad es la formación de profesionales que sean eficaces en el ejercicio de su profesión, contribuyendo al desarrollo científico, económico y social del país. Cumplir con este objetivo no es tarea fácil debido, principalmente, a la dificultad que arroja el crecimiento exponencial del saber en cualquiera de sus ramas, la falta de motivación de los estudiantes como consecuencia del marco social actual, y el carácter dinámico de nuestra sociedad, cuyo continuo cambio impide predecir cual será la formación que se exigirá a los profesionales en los próximos años. Estos factores han contribuido al cambio de orientación de las enseñanazas universitarias, que se han adaptado a las directrices del Espacio Europeo de Educación Superior (Sanabria-Codesal, 2014). Como consecuencia, los actuales planes de estudio y programaciones docentes se diseñan tomando como eje de referencia el propio aprendizaje de los alumnos. De esta manera la formación deja de entenderse como una simple transmisión de conocimientos, y se orienta hacia la adquisición de competencias que pretenden despertar en los alumnos actitudes receptivas y creativas que permitan desarrollar su capacidad de análisis y síntesis, imaginación, su iniciativa propia o capacidad para comunicarse y trabajar en equipo, aspectos altamente valorables en el perfil profesional de un Ingeniero Químico. El sector de la Industria Química, está influenciado tanto por el componente tecnológico como por el científico y sometido a un elevado ritmo de innovación. Existen más de 10 millones de sustancias químicas catalogadas e infinidad de procesos químicos (Heaton, 1996). Las empresas del sector reclaman profesionales cualificados capaces de adaptarse a las necesidades del momento, de conseguir información por sus propios medios, de comprenderla y criticarla, de crear e innovar.

La adquisición de estas competencias no es tarea sencilla y numerosos estudios en educación señalan la necesidad de emplear para ello metodologías activas que fomenten la participación del alumno en el proceso formativo (Olmedo, 2013). En general, el empleo de estas metodologías, entre las que se encuentran el aprendizaje colaborativo, cooperativo, basado en problemas, en casos de estudio o el aula inversa, conlleva una mejora significativa de los resultados de aprendizaje (Prince, 2004; Kember, 2005). El reto para los docentes radica en ampliar el repertorio metodológico intentando conocer bien $\mathrm{y}$ en profundidad las

(cc) EY-NC-ND 2018, Universitat Politècnica de València

Congreso IN-RED (2018) 
posibilidades de las diferentes estrategias e ir experimentado su aplicación en la práctica educativa (Fernández March, 2006).

El Máster en Ingeniería Química organizado conjuntamente por la Universidad Autónoma de Madrid y la Universidad Rey Juan Carlos, verificado en 2012 y modificado en 2017 por ANECA, ofrece al estudiante una formación avanzada para el ejercicio de la profesión de Ingeniero Químico. La estructura del plan de estudios se recoge en la Fig. 1 y toda la información relacionada con el título puede consultarse en el link del Máster Universitario en Ingeniería Química (Máster Universitario en Ingeniería Química). Se trata de un máster oficial con atribuciones profesionales, cuyas competencias se encuentran definidas en el Real Decreto 1393/2007 (básicas), 861/2010 (generales), 1027/2011 (transversales) y en la Resolución 12977 de 8 de junio de 2009, de la Secretaría General de Universidades (específicas).

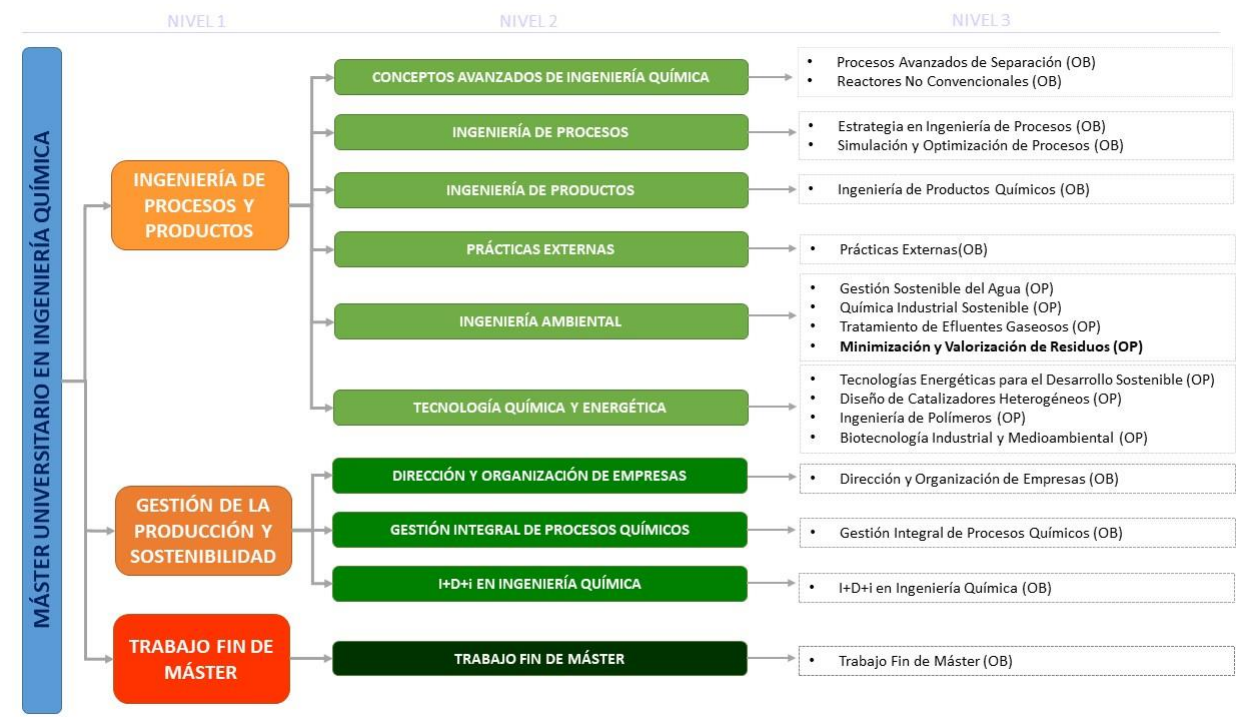

Fig. 1 Estructura del Máster en Ingeniería Química: NIVEL 1: módulos; NIVEL 2: materias; NIVEL 3: asignaturas. Se distinguen asignturas de carácter obligatorio $(O B)$ y optativo $(O P)$.

La asignatura de Minimización y Valorizacion de Residuos (MVR) es de carácter cuatrimestral, optativa, dotada con tres ECTS e impartida durante las primeras ocho semanas de curso. El número máximo de estudiantes está comprendido entre 25 y 30 . Los objetivos, competencias, contenidos, metodología y sistema de evaluación se recogen en la Guía Docente (Guía Docente de Minimización y Valorización de Residuos). El carácter optativo de la asignatura, el reducido número de estudiantes, su formación y su grado de madurez tras haber finalizado los estudios de grado, y el número de competencias adscritas a la asignatura (21) motivó al equipo docente a establecer diferentes metodologías activas para el desarrollo de la enseñanza, al cumplir la asignatura con los requisitos que garantizan el éxito de esta metodología (Fernández, 2016). 


\section{Objetivos}

El objetivo principal de este trabajo es la introducción de metodologías de aprendizaje activo y participativo que fomenten la adquisición de las competencias de la asignatura optativa Minimización y Valorización de Residuos del Máster en Ingeniería Química por la URJC y la UAM, centradas en la aplicación de los conocimientos adquiridos para al desarrollo de estrategias de gestión de residuos y de diseño de procesos de valorización de los mismos. Para ello, se han propuesto una serie de actividades docentes, coherentes con las competencias a adquirir, enmarcadas en metodologías de aprendizaje colaborativo, cooperativo, basado en problemas, en casos de estudio, aula inversa y docencia en red. La evaluación de la adquisición de las competencias como resultado de la implementación de esta metodología se realiza en base a las calificaciones obtenidas por los estudiantes (examen, evaluación frecuente y calificación global) en los primeros cuatro cursos de impartición en el máster.

Este trabajo nace de la convicción de que la enseñanza en el ámbito de la Minimización y Valorización de residuos debe tener lugar en situaciones lo más cercanas posibles al contexto profesional, con un nivel de dificultad y complejidad similares a los que los futuros egresados se encontrarán en la práctica profesional y, especialmente, bajo una actitud positiva de un aprendizaje activo útil para enfrentarse a problemas reales, aspecto que se evalúa de acuerdo al grado de satisfacción de los estudiantes con la asignatura.

\section{Desarrollo de la innovación}

\subsection{Presentación}

Tanto desde un punto de vista socioeconómico como técnico, el sector del tratamiento (y en particular de valorización) de residuos está en continuo crecimiento y constituye una fuente importante de empleo verde. Todo ello debe responder a las demandas de la sociedad, que percibe la generación de residuos como una de las mayores preocupaciones ambientales y como una responsabilidad individual y colectiva. El incremento en la generación de residuos a escala global es continuo y la prevención y correcta gestión son esenciales para evitar sus impactos negativos sobre el medio ambiente y la salud humana. Si hasta hace pocos años la valorización de residuos tenía como objetivo principal el cumplimiento de la normativa vigente, actualmente se está convirtiendo en una estrategia de negocio, lo que tiene una gran influencia al plantear el estudio técnico-económico de un proyecto de gestión de residuos. La relevancia del sector y sus expectativas de crecimiento hace necesario que se formen profesionales con capacidad para adaptarse a los retos que el desarrollo y la implantación de esta tecnología plantea y con capacidad para el auto aprendizaje a lo largo de la vida.

Bajo esa premisa, teniendo en cuenta que se trata de una asignatura optativa con una matrícula que oscila en torno a los 25 estudiantes y que se imparte en el último estadio de su formación, se diseñó e implementó un plan metodológico basado en el Aprendizaje Activo, que ha sido evaluado tras 4 años de implantación. Se realizó un riguroso análisis para seleccionar una

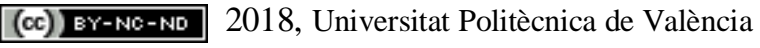

Congreso IN-RED (2018) 
amplia diversidad de actividades atendiendo a las competencias que deben adquirir los estudiantes al cursar esta signatura.

\subsection{Actividades formativas y adquisición de competencias}

Las competencias que los estudiantes deben adquirir tras cursar la asignatura se presentan en el Anexo I, e incluyen competencias básicas, generales, transversales y específicas. Para conseguir la adquisición de dichas competencias se han llevado a cabo las actividades formativas que se describen a continuación. En la Fig. 2 se presentan las actividades desarrolladas para cubrir cada una de las competencias de la asignatura.

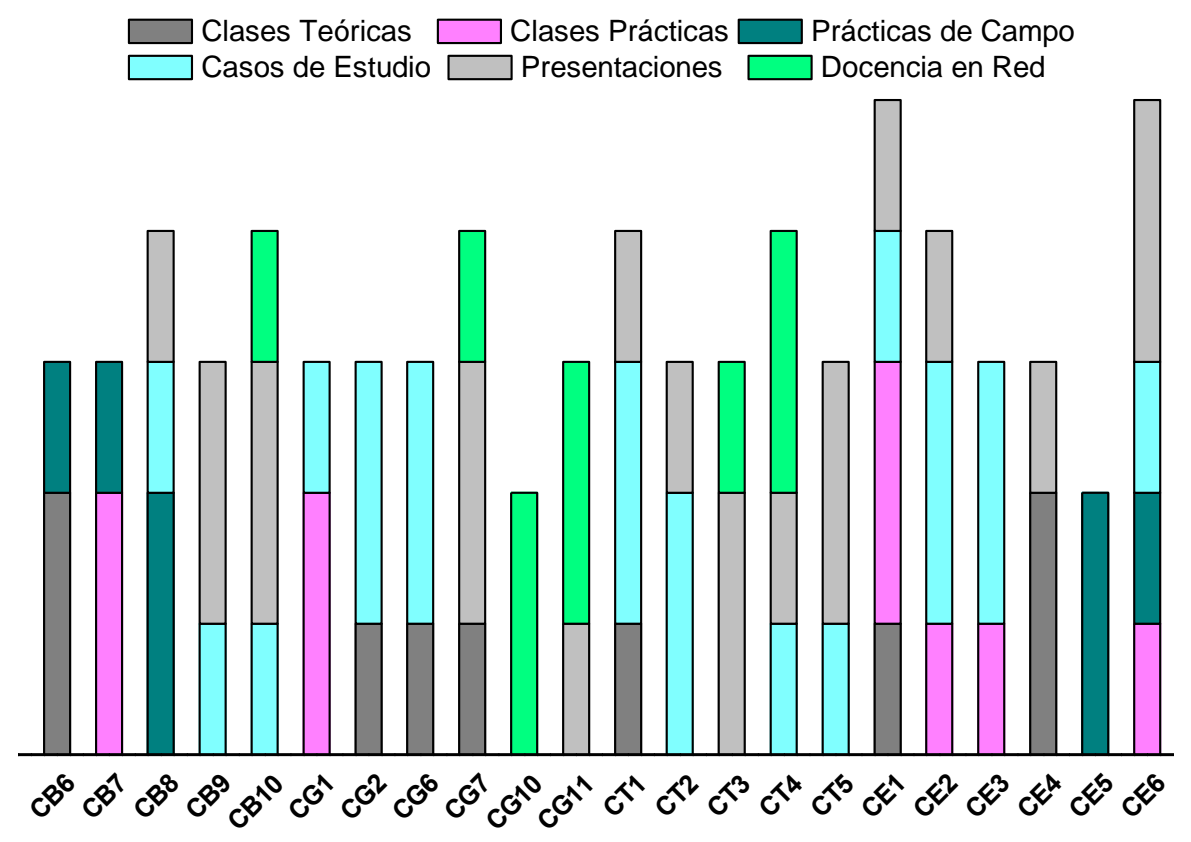

Fig. 2. Actividades formativas desarrolladas para fomentar la adquisión de las diferentes competencias.

\subsubsection{Actividades presenciales}

\section{Clases Teóricas en Aula}

Consisten en la exposición por parte del profesor de los contenidos fundamentales de la mayor parte de los temas. Se han diseñado para presentar los aspectos básicos de la gestión de residuos y su marco legislativo así como los conceptos científico-técnicos básicos de diferentes operaciones de valorización y los aspectos más relevantes de la tecnología implantada. En cada tema se establece una relación estudiante - profesor a través de la dinámica "preguntas y respuestas" para estimular el razonamiento crítico y la comprensión de las materias. 
Las clases teóricas en aula proporcionan conocimientos a los estudiantes que favorecen el desarrollo y aplicación de ideas, la solución de problemas y facilita el autoaprendizaje posterior ya que orienta sobre los contenidos a desarrollar.

\section{Clases prácticas en Aula}

Consisten en la preparación, resolución y/o discusión de problemas o casos de estudios: se proporcionan a los estudiantes problemas para resolver y se plantean temas de debate a partir de noticias actuales en temática de residuos. Se realizan tanto de forma individual como en grupo.

La resolución de problemas se ha considerado fundamental en la enseñanza de ciencias experimentales, de modo que no se podría entender su estudio sin demostrar que la teoría funciona. La didáctica de las ciencias experimentales conlleva implícitamente la habilidad de resolución de problemas (Pérez Bernabeú, 2016). La actividad profesional del Ingeniero Químico se centra principalmente en la práctica, por lo que la resolución de problemas resulta clave para hacer de puente entre el mundo académico y empresarial.

A través de esta actividad los estudiantes tienen que aplicar los conocimientos adquiridos y demuestran su capacidad para resolver problemas y establecer soluciones viables. Se trabajan competencias básicas, generales y específicas como la CB7, CG1 y CE1.

\section{Prácticas de Campo}

Se realizan dos actividades diferentes:

i) Visita a una instalación representativa del sector.

ii) Seminario impartido por un profesional en gestión de residuos.

Estas actividades tienen una gran importancia ya que en la mayoría de los casos suponen un primer contacto con el mundo empresarial de la gestión de residuos. Ofrecen la posibilidad de intercambiar conocimientos y experiencias con profesionales expertos en el área y conocer de forma directa procesos reales y los retos asociados al sector. Ambas actividades pretender proporcionar a los estudiantes una visión integral de la gestión de residuos dentro de un ámbito profesional concreto. Potencian competencias básicas y específicas como la CB8 y CE5.

\subsubsection{Actividades dirigidas}

\section{Casos de estudio}

Se plantean dos tareas dentro del epígrafe casos de estudios:

i) Planteamiento de una estrategia de gestión de residuos urbanos.

ii) Análisis de literatura científica relacionada con nuevas tecnologías para la valorización de residuos.

Con ellas se pretende, por una parte, potenciar la aplicación e integración de conocimientos que permitan diseñar una estrategia de gestión de residuos, y por otra, fomentar al estudiante el aprendizaje autónomo a partir de artículos científicos que les permitan el desarrollo continuo de su profesión.

(cc) EY-NC-ND 2018, Universitat Politècnica de València

Congreso IN-RED (2018) 
En el primer caso los estudiantes, mediante trabajo en grupo, tienen que plantear una estrategia para el tratamiento de un residuo y realizar un diseño básico de las operaciones de valorización seleccionadas. Es un caso totalmente abierto, que favorece la toma de decisiones, con sus consecuencias posteriores, ya que tienen la posibilidad de seleccionar la estrategia de tratamiento del residuo entre todas las alternativas disponibles. Lleva implícita la necesidad de discusión de las alternativas entre los integrantes del grupo y la de llegar a un consenso. Por otra parte, en función de la estrategia seleccionada tienen que plantear sus propios casos prácticos que posteriormente tendrán que resolver. Esto les ayuda a trabajar la primera y decisiva fase en la resolución de problemas que consiste en entender y explorar el problema (Polya, 2014). El cambio de rol, en el que el alumno pasa de resolver problemas a plantear los problemas que posteriormente ha de resolver, favorece el desarrollo de las habilidades del estudiante para enfrentarse a la resolución de problemas complejos.

La segunda actividad ofrece al estudiante la posibilidad de explorar tecnologías emergentes y abrir el horizonte de las posibilidades que ofrece el mundo de la valorización de residuos y su potencial para el beneficio de la sociedad y del medio ambiente y para sus posibilidades profesionales.

Esta actividad, junto con la de aula inversa tienen una gran relevancia ya que concentran la mayor parte de las competencias transversales y una buena parte de las competencias específicas de la asignatura. Se trabajan con intensidad las competencias CT1, CT2, CE2 y CE3.

\section{Presentaciones (Aula inversa)}

Mediante esta actividad los estudiantes elaboran y exponen un tema perteneciente al bloque "Valorización de residuos municipales, agrícolas e industriales". Esta actividad se realiza en grupo (3-4 estudiantes). Los temas a desarrollar son ofertados por el equipo docente y cambian cada curso académico. Los docentes proponen el esquema metodológico a seguir para el desarrollo de cada tema. Los estudiantes realizan la búsqueda de los contenidos, los seleccionan y organizan. Después realizan un documento de presentación y lo exponen en clase, con participación de todos los integrantes del grupo. De este modo, se utilizan metodologías de aprendizaje cooperativo y de cambio de roles ya que en este caso son los estudiantes los que preparan y exponen los temas. Se potencian, entre otras, competencias transversales como el trabajo en equipo, comunicación oral, aprendizaje autónomo y otras habilidades interpersonales (argumentación, consenso, negociación, etc..). Éstas son competencias en las que los recién titulados de grado presentan carencias, necesarias para el desempeño profesional en la sociedad actual, y que se adquieren con la práctica (Domingo, 2008; Chica, 2011), por lo que resulta más eficiente practicarlas en un máster que prepara especialistas con una formación avanzada y orientada a la especialización profesional (Ureña, 2012, Trigueros, 2016). Fomenta además la integración de conocimientos, emisión de juicios y toma de decisiones. Fomenta la adquisición de un importante número de competencias (Fig. 2), y de forma intensiva las CB9, CB10, CT3, CT5 y CE6.

\section{Docencia en red}

Se proporciona abundante material didáctico: legislación, planes estratégicos, información sobre instalaciones de gestión de residuos, artículos científicos, vídeos. El material de apoyo colgado en la plataforma Moodle pretende fomentar en los estudiantes el aprendizaje 
autónomo y la adaptación a los cambios y progresos a los que deberán enfrentarse como titulados Máster en Ingeniería Química.

\subsection{Evaluación del grado de adquisición de competencias}

El grado de adquisición de las competencias desarrolladas por el estudiante se determina mediante una evaluación frecuente (50-60\% de la nota final) y un examen (40-50\% de la nota final). La evaluación frecuente incluye: participación activa en las clases teóricas y prácticas, seminarios y prácticas de campo (10\% de la calificación final), entrega de casos prácticos (10-20\%) y presentaciones (20-30\%). De esta forma se asegura la evaluación de los resultados de aprendizaje en base a todas las competencias trabajadas.

La actitud de los estudiantes hacia la aplicación de metodologías de aprendizaje activo, aspecto clave para enfrentarse a la resolución de problemas reales, se evalúa de acuerdo al grado de satisfacción de los estudiantes con la asignatura. Éste queda recogido en el Informe Anual de Garantía de Calidad que la titulación elabora cada curso académico. En este Informe se resumen los resultados de las encuestas de satisfacción de los estudiantes con cada asignatura del Máster.

\section{Resultados}

Las actividades formativas planificadas en la asignatura MVR: Clases Teóricas, Clases Prácticas, Prácticas de Campo, Casos de Estudio, Presentaciones y Docencia en Red, integran todas las competencias que el estudiante debe adquirir durante el curso (Fig. 2). Como se observa en esta Fig. 2, las competencias CE1 y CE6 son las que más se fomentan por ser consideradas las más importantes para el desempeño profesional ya que atienden a la participación activa en la resolución de problemas de carácter técnico. Por el contrario, las competencias CG10 y CE5 son las que menos se desarrollan debido a que requiere que el estudiante se encuentre integrado en un contexto profesional real.

La Fig. 3 muestra que las actividades formativas Casos de Estudio y Presentaciones, son las que permiten el desarrollo (parcial) de un mayor número de competencias. Por esta razón, estas dos actividades formativas tienen un peso importante en la calificación final de la asignatura (hasta el 30\%). Sin embargo, hay que tener en cuenta, que aunque se trata de actividades colaborativas que les hace aprender mejor los contenidos gracias a las ayudas de sus iguales (Gómez Mújica, 2003), los estudiantes de un mismo grupo tienden a adquirir roles, siendo unos más activos que otros (Torrelles, 2011). Por este motivo, se contempla la evaluación individual en forma de examen escrito que supone el 40-50\% de la calificación final de la asignatura. El objetivo de esta prueba, que comprende una parte teórica y otra práctica, es conocer en qué grado los estudiantes han interiorizado los contenidos de la asignatura.

(cc) EY-NC-ND 2018, Universitat Politècnica de València

Congreso IN-RED (2018) 


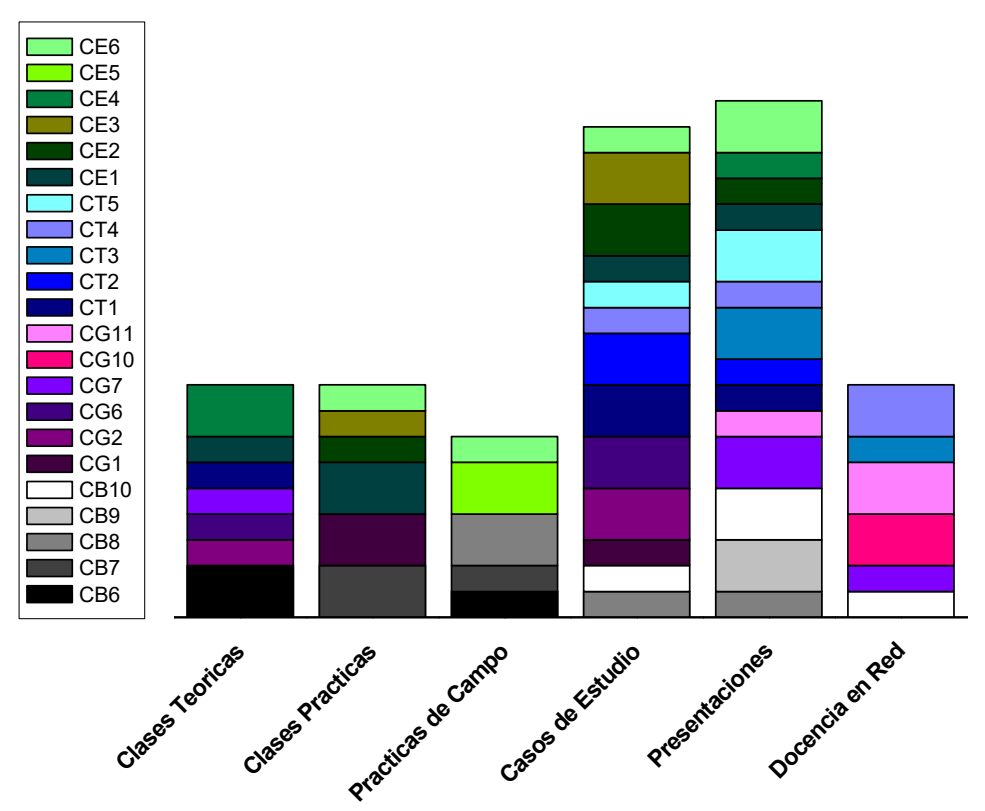

Fig. 3. Distribución ponderada de las competencias en las distintas actividades formativas aplicadas

Con el fin de valorar el grado de aprendizaje adquirido por los estudiantes en las competencias evaluadas en la asignatura MVR mediante la metodología basada en el aprendizaje activo y participativo se han analizado las calificaciones del examen, la evaluación frecuente y calificaciones globales obtenidas en los cuatro años académicos de impartición de la asignatura. En la Fig. 4 se aprecia que las calificaciones obtenidas en la evaluación frecuente son altas, comprendidas entre el notable y sobresaliente (8-9,3), es decir, que los estudiantes alcanzan un mayor grado de aprendizaje en las competencias adquiridas mediante estas actividades (Fig. 2 y 3). Se ha observado positivamente, que los estudiantes llegan a involucrarse y comprometerse más en la asignatura al responsabilizarse de su propio aprendizaje, siendo capaces de resolver los casos prácticos de manera adecuada, algunos incluso con simulaciones muy creativas y realizando, en general, unas presentaciones orales estructuradas y bien explicadas.

Por otro lado, la evaluación mediante examen escrito que incluye análisis, reflexión y justificación no supone una mejora en las calificaciones de la asignatura (Fig. 4). Ésta se realiza en un contexto más alejado de la práctica profesional ya que se trata de una prueba individual, sin intercambio de ideas y coordinación de esfuerzos, y sin apoyo de bases bibliográficas. Sin embargo, la nota media del examen es bastante alta lo que demuestra que la aplicación de estas metodologías favorece el aprendizaje global de la asignatura. Además, existe una tendencia de mejora de la calificación del examen con la evolución de la asignatura en el transcurso de los cursos, probablemente debido a la mayor experiencia y asentamiento de la metodología activa. 


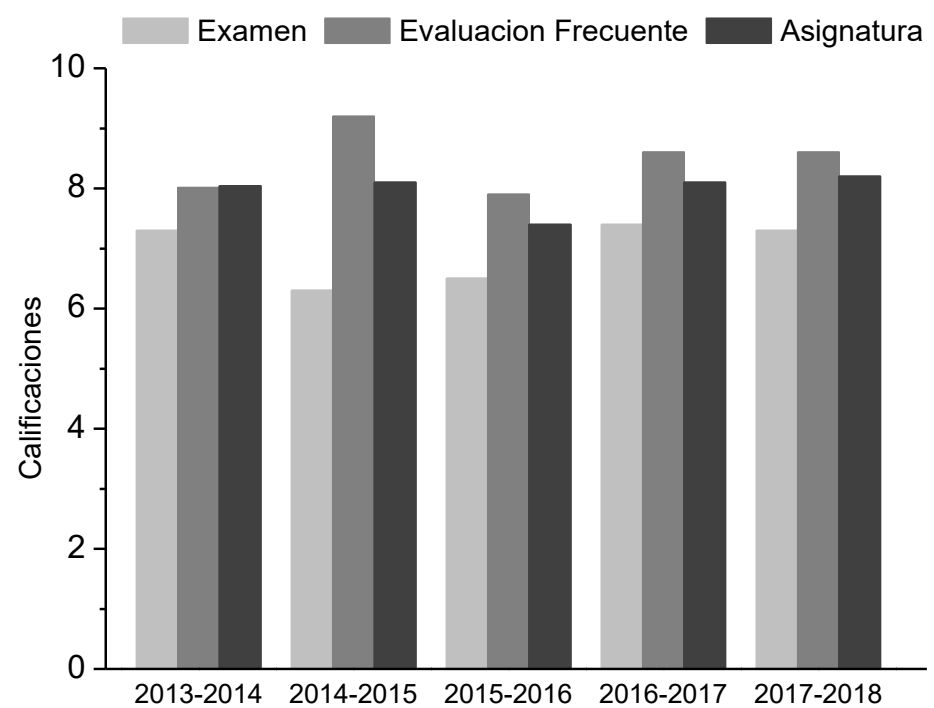

Fig. 4. Calificaciones promedio del examen escrito, de las actividades formativas (evaluación frencuente) y de la asignatura obtenidas en los diferentes cursos académicos

Otro aspecto interesante es el grado de satisfacción de los estudiantes con la asignatura. En la Fig. 5 se aprecia que es una asignatura con una elevada valoración, comprendida entre 4,3 y 4,6 sobre 5. Ésta es superior al grado de satisfacción promedio de todas las asignaturas optativas del máster y del máster en sí. Este grado de aceptación se puede comprobar también por el número de matriculados en la asignatura, siempre correspondiente a la capacidad máxima de matrícula (25-30 estudiantes, dependiendo del número de estudiantes matriculados en el máster cada curso académico).

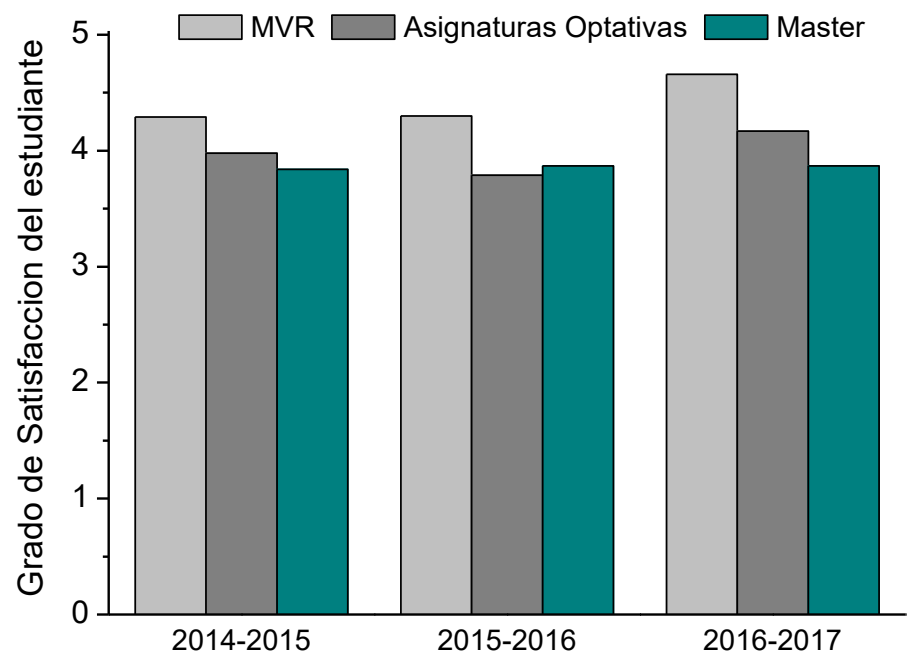

Fig. 5. Grado de satisfacción de los estudiantes en el Máster en Ingeniería Química por la URJC y UAM de la asignatura MVR y valoracion promedio de las asignaturas optativas y del máster. 


\section{Conclusiones}

Los resultados obtenidos en la aplicación de diversas metodologías de aprendizaje activo enfocadas a promover la adquisición de las competencias atribuidas a la asignatura de Minimización y Valorización de Residuos fueron satisfactorios. Los alumnos demostraron haber adquirido un aprendizaje extenso en la materia, destacando la calificación obtenida en las actividades de evaluación frecuente (8-9,3), en la que tiene mayor peso las actividades en las que el estudiante tiene un papel protagonista en su aprendizaje y que desarrollan además un mayor número de competencias. Estas metodologías favorecen la adquisición e integración de los conocimientos, lo que se refleja en los buenos resultados de la calificación global. Por otra parte, la metodología aplicada fomentó el compromiso y motivación de los estudiantes siendo la valoración de la asignatura por parte de los mismos elevada y superior a la media del máster y de las otras asignaturas optativas.

\section{Referencias}

CHICA, E. (2011) “Una propuesta de evaluación para el trabajo en grupo mediante rúbrica." Escuela Abierta, 2011, 14, 67-81

DOMINGO, J (2008) “El aprendizaje cooperativo" Cuadernos de Trabajo Social, Vol. 21 (2008): 231246

España. Real Decreto 1393/2007, de 29 de octubre, por el que se establece la ordenación de las enseñanzas universitarias oficiales. BOE, 30 de octubre de 2007, núm. 260, p. 1-28.

España. Resolución de 8 de junio de 2009, de la Secretaría General de Universidades, por la que se da publicidad al Acuerdo del Consejo de Universidades, por el que se establecen recomendaciones para la propuesta por las universidades de memorias de solicitud de títulos oficiales en los ámbitos de la Ingeniería Informática, Ingeniería Técnica Informática e Ingeniería Química. BOE, 4 de agosto de 2009, núm. 187, p. 66699-66710.

España. Real Decreto 861/2010, de 2 de julio, por el que se modifica el Real Decreto $1393 / 2007$, de 29 de octubre, por el que se establece la ordenación de las enseñanzas universitarias oficiales. BOE, 3 de julio de 2010, núm. 161, p. 58454-58468.

España. Real Decreto 1027/2011, de 15 de julio, por el que se establece el Marco Español de Cualificaciones para la Educación Superior. BOE, 3 de agosto de 2011, núm. 185, p. 8791287918.

FERNÁNDEZ, M.S., GARCÍA-FAYOS, B. y ARNAL ARNAL J.M. (2016) “Análisis de la mejora del aprendizaje tras la incorporación de metodologías activas en una asignatura optativa del Grado de Ingeniería Química" En : Congreso Nacional de Innovación Educativa y de Docencia en Red (julio, 2016, Valencia). Valencia : Editorial Universitat Politécnica de València.

FERNÁNDEZ MARCH, A. (2006) "Metodologías activas para la formación de competencias" En Educatio siglo XXI, 24, 35-56.

Guía Docente de Minimización y Valorización de Residuos. <http://www.uam.es/ss/Satellite/Ciencias les/1242655139058/listadoGuiasDocentes/Estudios de Posgrado.htm> [Consulta: 23 de marzo de 2018].

GOMEZ MUJICA, A. y ACOSTA RODRIGUEZ, H. (2003). « Acerca del trabajo en grupos o equipos ». ACIMED, http://eprints.rclis.org/5035/1/acerca.pdf .

HEATON, A. (1996) “An introduction to Industrial Chemistry” Chapman \& Hall. London.

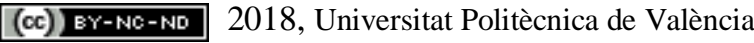

Congreso In-Red (2018) 
Aplicación de metodologías de aprendizaje activo para promover la adquisición de competencias en la asignatura Minimización y Valorización de Residuos

KEMBER, D., LEUNG, D.Y.P. (2005) "The influence of active learning experiences on the development of graduate capabilities" En : Studies in Higher Education, 30, 2, 155-170.

Máster Universitario en Ingeniería Química.

http://www.uam.es/ss/Satellite/es/1242684629435/1242669469071/masteroficial/masterOficia/Master Universitario en Ingenieria Quimica.htm> [Consulta: 23 de marzo de 2018].

OLMEDO, E. M. (2013). "Enfoques de aprendizaje de los estudiantes y metodología docente: Evolución hacia el nuevo sistema de formación e interacción propuesta en el EEES." Revista de Investigación Educativa, 31, 2, 411-429.

PÉREZ-BERNABEU, E., SELLÉS, M.A., GISBERT, V., SÁNCHEZ-CABALLERO, S. (2016) “Un enfoque diferente para la resolución de problemas." En : Congreso Nacional de Innovación Educativa y de Docencia en Red (julio, 2016, Valencia). Valencia : Editorial Universitat Politécnica de València POLYA, G. (2014). How to Solve It: A New Aspect of Mathematical Method. Princeton University Press.

PRINCE, M. (2004) "Does active learning work ? A review of the research." En : Journal of Engineering Education, 93, 3, 223-231.

SANABRIA-CODESAL, E., BOSCH, I., VICENT, M.C., LLORET, J., ALVAREZ, S., ROMERO, L (2014) "Análisis de las Dimensiones Competenciales Incluidas en Diferentes Asignaturas en Ingenierías" En: Jornadas de Innovación Educativa y de Docencia en Red (julio, 2014, Valencia). Valencia : Universidad Politécnica de Valencia.

TORRELLES C., COIDURAS J., ISUS S., CARRERA F. X. PARIS G., CELA J. M. (2011) "Competencia de Trabajo en equipo: definición y categorización. Profesorado". Revista de Currículum y Formación de Profesorado [online].

TRIGUEROS, S., PEREZ, D., SOLANA, P. y GARCIA, E. (2016) "Experiencia docente universitaria mediante el uso de metodologías activas para el aprendizaje como la evaluación formativa compartida y el trabajo de competencias transversales." En : Congreso Nacional de Innovación Educativa y de Docencia en Red (julio, 2016, Valencia). Valencia : Editorial Universitat Politécnica de València

UREÑA, N y RUIZ, E. (2012). "Experiencia de evaluación formativa y compartida en el Máster Universitario en Formación del Profesorado de Educación Secundaria" Psychology, Society \& Education, 4, 1, 29-44.

\section{Anexo I. Competencias asignadas a la asignatura}

Competencias Básicas (según Real Decreto 1393/2007, de 29 de octubre)

CB6 - Poseer y comprender conocimientos que aporten una base u oportunidad de ser originales en el desarrollo y/o aplicación de ideas, a menudo en un contexto de investigación.

CB7 - Que los estudiantes sepan aplicar los conocimientos adquiridos y su capacidad de resolución de problemas en entornos nuevos o poco conocidos dentro de contextos más amplios (o multidisciplinares) relacionados con su área de estudio.

CB8 - Que los estudiantes sean capaces de integrar conocimientos y enfrentarse a la complejidad de formular juicios a partir de una información que, siendo incompleta o limitada, incluya reflexiones sobre las responsabilidades sociales y éticas vinculadas a la aplicación de sus conocimientos y juicios.

CB9 - Que los estudiantes sepan comunicar sus conclusiones -y los conocimientos y razones últimas que las sustentan- a públicos especializados y no especializados de un modo claro y sin ambigüedades.

CB10 - Que los estudiantes posean las habilidades de aprendizaje que les permitan continuar estudiando de un modo que habrá de ser en gran medida autodirigido o autónomo.

Competencias generales (según Real Decreto 861/2010, de 2 de julio)

CG1 - Capacidad para aplicar el método científico y los principios de la ingeniería y economía, para formular y resolver problemas complejos en procesos, equipos, instalaciones y servicios, en los que la materia experimente cambios en su composición, estado o contenido energético, característicos de la industria química y de otros sectores

(cc) EY-NC-ND 2018, Universitat Politècnica de València

Congreso IN-RED (2018) 
relacionados entre los que se encuentran el farmacéutico, biotecnológico, materiales, energético, alimentario o medioambiental.

CG2 - Concebir, proyectar, calcular, y diseñar procesos, equipos, instalaciones industriales y servicios, en el ámbito de la ingeniería química y sectores industriales relacionados, en términos de calidad, seguridad, economía, uso racional y eficiente de los recursos naturales y conservación del medio ambiente.

CG6 - Tener capacidad de análisis y síntesis para el progreso continuo de productos, procesos, sistemas y servicios utilizando criterios de seguridad, viabilidad económica, calidad y gestión medioambiental.

CG7 - Integrar conocimientos y enfrentarse a la complejidad de emitir juicios y toma de decisiones, a partir de información incompleta o limitada, que incluyan reflexiones sobre las responsabilidades sociales y éticas del ejercicio profesional.

CG10 - Adaptarse a los cambios, siendo capaz de aplicar tecnologías nuevas y avanzadas y otros progresos relevantes, con iniciativa y espíritu emprendedor.

CG11 - Poseer las habilidades del aprendizaje autónomo para mantener y mejorar las competencias propias de la ingeniería química que permitan el desarrollo continuo de la profesión.

Competencias transversales (según Real Decreto 1027/2011, de 15 de julio)

CT1 - Adquirir conocimientos avanzados y demostrar, en un contexto de investigación científica y tecnológica o altamente especializado, una comprensión detallada y fundamentada de los aspectos teóricos y prácticos y de la metodología de trabajo en el campo de la Ingeniería Química.

CT2 - Saber aplicar e integrar los conocimientos, la comprensión de estos, su fundamentación científica y sus capacidades de resolución de problemas en entornos nuevos y definidos de forma imprecisa, incluyendo contextos de carácter multidisciplinar tanto investigadores como profesionales altamente especializados.

CT3 - Saber evaluar y seleccionar la teoría científica adecuada y la metodología precisa de sus campos de estudio para formular juicios a partir de información incompleta o limitada incluyendo, cuando sea preciso y pertinente, una reflexión sobre la responsabilidad social o ética ligada a la solución que se proponga en cada caso.

CT4 - Ser capaces de predecir y controlar la evolución de situaciones complejas mediante el desarrollo de nuevas e innovadoras metodologías de trabajo adaptadas al ámbito científico/investigador, tecnológico o profesional concreto, en general multidisciplinar, en el que se desarrolla la actividad de un titulado con el Máster Universitario en Ingeniería Química.

CT5 - Ser capaces de asumir la responsabilidad de su propio desarrollo profesional y de su especialización en el campo de la Ingeniería Química.

Competencias específicas (según Resolución 12977 de 8 de junio de 2009, de la Secretaría General de Universidades)

CE1 - Aplicar conocimientos de matemáticas, física, química, biología y otras ciencias naturales, obtenidos mediante estudio, experiencia, y práctica, con razonamiento crítico para establecer soluciones viables económicamente a problemas técnicos.

CE2 - Diseñar productos, procesos, sistemas y servicios de la industria química, así como la optimización de otros ya desarrollados, tomando como base tecnológica las diversas áreas de la ingeniería química, comprensivas de procesos y fenómenos de transporte, operaciones de separación e ingeniería de las reacciones químicas, nucleares, electroquímicas y bioquímicas.

CE3 - Conceptualizar modelos de ingeniería, aplicar métodos innovadores en la resolución de problemas y aplicaciones informáticas adecuadas, para el diseño, simulación, optimización y control de procesos y sistemas.

CE4 - Tener habilidad para solucionar problemas que son poco familiares, incompletamente definidos, y tienen especificaciones en competencia, considerando los posibles métodos de solución, incluidos los más innovadores, seleccionando el más apropiado, y poder corregir la puesta en práctica, evaluando las diferentes soluciones de diseño. CE5 - Dirigir y supervisar todo tipo de instalaciones, procesos, sistemas y servicios de las diferentes áreas industriales relacionadas con la ingeniería química.

CE6 - Diseñar, construir e implementar métodos, procesos e instalaciones para la gestión integral de suministros y residuos, sólidos, líquidos y gaseosos, en las industrias, con capacidad de evaluación de sus impactos y de sus riesgos. 